\title{
World watching as troubling new type of avian influenza surfaces in China
}

A subtype of avian influenza that has never before infected humans - H7N9 - continues to cause a steady stream of cases in China as international experts converged on Shanghai and Beijing this past week.

Canadian and World Health Organization (WHO) experts are cautious in characterizing the outbreak because many aspects of it remain unclear, including how the infection was acquired in the 102 cases confirmed as of Apr. 21. The virus was discovered in humans just three weeks earlier.

"Evidence suggests that poultry is a vehicle of transmission. But epidemiologists haven't yet been able to establish a strong and clear link," remarked Dr. Michael O'Leary, leader of a WHO joint mission of more than 15 Chinese and international experts, in a briefing held at the outset of the mission, which ran from Apr. 19 to 23. "Only a handful of the tens of thousands of chicken and birds tested have been positive for H7N9. We are still uncertain about the source of illness in people."

According to David Kelvin, a senior scientist at Toronto General Research Institute in Ontario who is currently working in China, $40 \%$ of patients with severe symptoms have reported contact with poultry, but another $40 \%$ had no such contact, suggesting an as-yet unidentified source of transmission.

These aspects differ from the H5N1 avian influenza subtype that has caused localized outbreaks over the past 15 years, says Dr. Allison McGeer, a microbiologist and director of infectious diseases at Mount Sinai Hospital in Toronto. By contrast, H7N9 "is a low pathogenic virus in birds, so it does not cause bird illness. It has caused a substantial number of human cases in a short period of time. And it is not clear that the sporadic human cases have been linked to poultry."

Whether the virus is being transmit-

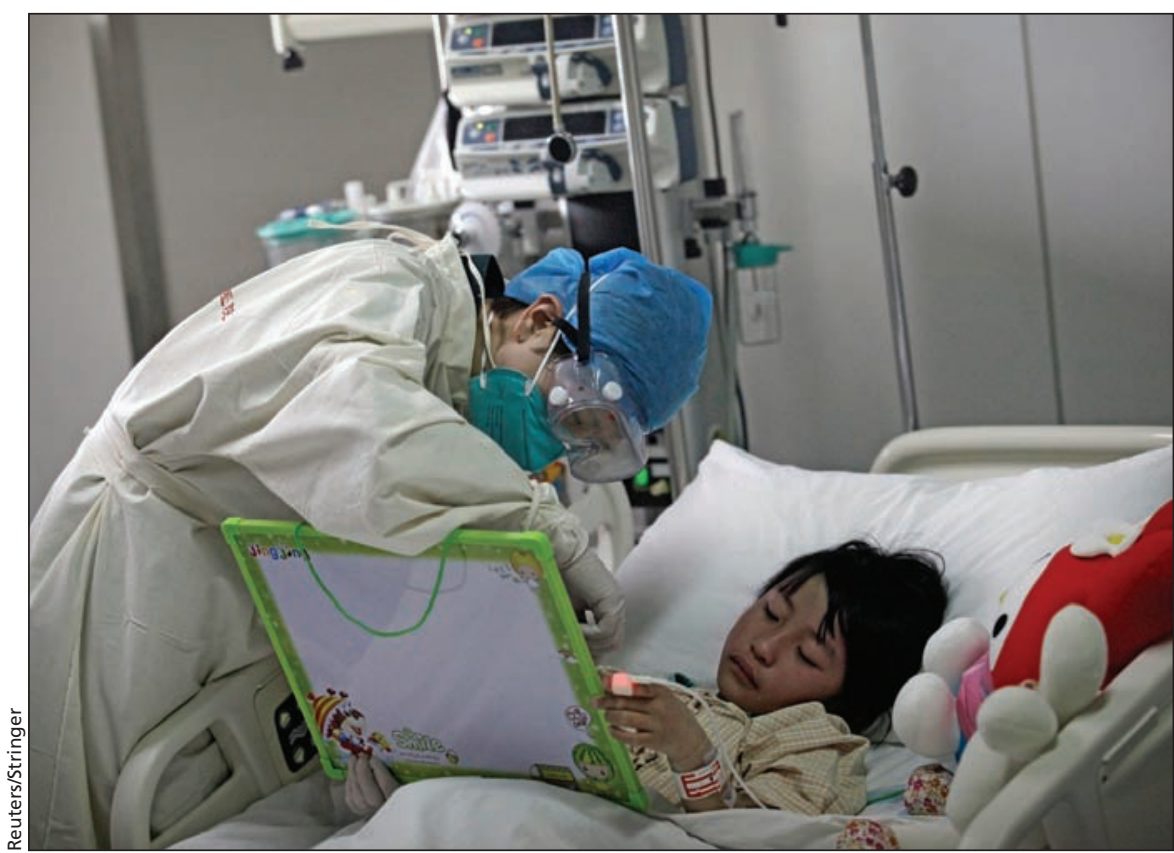

A seven-year-old girl infected with H7N9 avian flu is treated at Ditan Hospital in Beijing China. She was the first case to be reported outside the Yangtze River delta in East China where the new strain emerged in March.

ted among humans is also unclear. While there have been three family clusters of cases, "it's extremely difficult to sort out whether one gave it to the other, or they both got it from the same source," O'Leary said. "These clusters are of concern, obviously, for investigation, because of the possibility that they represent human-to-human transmission." If so, "this is the very limited, rare sort of human-to-human transmission, rather than the easy and sustained transmission that emerges into a human pandemic virus."

Kelvin agrees the family clusters indicate that "human-to-human transmission cannot be ruled out, and the evidence suggests it is possible." However, he says thousands of patient contacts who have been followed and tested show no signs of infection.

McGeer, who sits on national and provincial infectious disease and pandemic influenza committees, explains that "it is clear that some avian strains are in some circumstances transmitted from one human being to another but they don't make it to be a pandemic. We don't know the on/off switch for pandemics."

As well, the mortality rate is unclear. While $20 \%$ of identified patients have died, "until we have a population-based sample of cases you really can't judge the mortality rate."

H7- and H5-group influenza viruses have characteristics that are of particular concern in birds. "It is known in the scientific community that $\mathrm{H} 7$ and $\mathrm{H} 5$ viruses are prone to mutate," explains Dr. Abed Harchaoui, senior staff veterinarian in the Foreign Animal Disease Section of the Canadian Food Inspection Agency.

H7 and H5 subtypes normally circulate in wild birds and may be transmitted to domestic poultry such as chickens and turkeys in several ways. "It can be contact between wild birds and poultry through free-range farming," explains Harchaoui, "Also from 
excretion from the birds from outside to inside of the barn. People can step in it and get it on their boots."

"In some situations, like ducks and geese, the virus is low pathogenic, but might mutate very quickly in chickens and turkeys," becoming highly pathogenic and causing severe symptoms and death.

This was shown dramatically in an outbreak of a related influenza, H7N3, in a chicken farm in the Fraser Valley of British Columbia in 2004. The virus had caused only decreased egg production and a slight uptick in mortality in a barn on the farm. But an outbreak 10 weeks later in a second barn caused a spike in mortality, because of a genetic mutation occurring between one barn and the other (Rev Sci Tech 2009;28: 349-358).

McGeer says the ability of $\mathrm{H} 7$ viruses to mutate quickly and move from low to high pathogenicity adds to the concern about infection in humans.

There have been previous, although rare, H7-group influenza outbreaks in humans, such as an H7N7 outbreak in the Netherlands a decade ago, which was "rapidly and effectively managed," says McGeer. It remains unclear what course this outbreak will take. "All we can do is wait and see." - Carolyn Brown, Ottawa, Ont.

CMAJ 2013. DOI:10.1503/cmaj.109-4477 